\title{
ANALYTIC CONTINUTATION OF ARCHIMEDEAN WHITTAKER INTEGRALS
}

\author{
STEPHEN RALLIS AND DAVID SOUDRY
}

(Communicated by Larry J. Goldstein)

\begin{abstract}
We prove the analytic continuation of a certain family of Whittaker Archimedean integrals that arise as local factors of global $L$-functions associated to the standard representation of certain classical groups.
\end{abstract}

\section{INTRODUCTION}

In this paper we use a variation of Wallach [W] to prove the meromorphic continuation of a certain type of integrals. Our motivating example comes from the construction in [PS-R] generalizing Andrianov's construction of the $L$-function for Siegel modular forms [A]. The work in [PS-R] also explains the local-global structure of Andrianov's proof by introducing a new principle which enables us to get Euler products in some cases when there is no uniqueness of some appropriate "Whittaker model". In this paper we are concerned with the meromorphic continutation (in the Archimedean case) of integrals of such type as those encountered in [PS-R]. Let us review the basic construction in [PS-R] so that we can keep this example in mind.

Let $G=\mathrm{Sp}(n)$, the symplectic group of rank $n$, regarded as an algebraic over $\mathbf{Q}$ (we take $\mathbf{Q}$ instead of a global field just for the sake of simplicity). Assume that $n$ is even. Let $T \in M(n, \mathbf{Q})$ be symmetric and nondegenerate, and $O_{T}$ the orthogonal group of $T$. Consider the oscillator representation $\omega$, which corresponds to the reductive dual pair $\left(O_{T}, \mathrm{Sp}(n)\right)$ and a fixed nontrivial character $\psi$ of $\mathbf{Q} \backslash \mathbf{A}$ ( $\mathbf{A}$ is the ring of adeles of $\mathbf{Q}$ ); $\omega$ may be realized on $S(M(n, \mathbf{A}))$, the space of Schwartz-Bruhat functions on $M(n, \mathbf{A})$. For $\phi$ in $S(M(n, \mathbf{A}))$, consider the $\theta$-series

$$
\theta_{T}^{\phi}(g)=\sum_{x \in M(n, \mathbf{Q})} \omega(g) \phi(x), \quad g \in G(\mathbf{A}) .
$$

Received by the editors March 15, 1987 and, in revised form, Feburary 23, 1988.

1980 Mathematics Subject Classification (1985 Revision). Primary 10D40; Secondary 10D12.

Both authors were supported by US-Israel Binational Grant 84-00139. The first author was also supported by NSF grant DMS-8401947. 
Let $P$ be the parabolic subgroup $\left({ }^{*} *\right)$ of $G$, and let

$$
E(g, s)=\sum_{\gamma \in P(\mathbf{Q}) \backslash G(\mathbf{Q})} f(\gamma g, s)
$$

be a properly normalized Eisenstein series corresponding to the representation $\operatorname{Ind}_{P(\mathbf{A})}^{G(\mathbf{A})}|\operatorname{det}|^{s+n+1 / 2}$. See [PS-R] for the precise normalization. Let $\pi$ be an irreducible, automorphic, cuspidal representation of $G(\mathbf{A})$. Consider for a cusp form $\varphi$ in the space of $\pi$, the integral

$$
\int_{G(\mathbf{Q}) \backslash G(\mathbf{A})} \varphi(g) \theta_{T}^{\phi}(g) E(g, s) d g .
$$

For $\operatorname{Re}(s)$ large enough, this integral equals

$$
I(s)=\int_{N(\mathbf{A}) \backslash G(\mathbf{A})} \varphi_{T}(g) \omega(g) \phi\left(I_{n}\right) f(g, s) d g .
$$

Here $N$ is the unipotent radical of $P$ and

$$
\varphi_{T}(g)=\int_{\operatorname{Sym}^{n}(\mathbf{Q}) \backslash \operatorname{Sym}^{n}(\mathbf{A})} \psi^{-1}(\operatorname{tr} T X) \varphi\left(\left(\begin{array}{cc}
I_{n} & X \\
0 & I_{n}
\end{array}\right) g\right) d X .
$$

Let $S$ be a large enough finite set of primes, containing $\infty$, outside of which all the data of $I(s)$ is "unramified". Let $\Omega$ be a finite set of primes of $\mathbf{Q}$ containing $S$. Put

$$
\begin{aligned}
I_{\Omega}(s) & =\int_{N\left(\mathbf{A}_{\Omega}\right) \backslash G\left(\mathbf{A}_{\Omega}\right)} \varphi_{T}(g) \omega(g) \phi\left(I_{n}\right) f(g, s) d g, \\
J(s) & =I_{S}(s) .
\end{aligned}
$$

Let $\phi=\bigotimes \phi_{p}, f=\bigotimes f_{p}$; then it is proved that for a prime $p$ not in $\Omega$

$$
I_{\Omega U\{p\}(s)}=L\left(\pi_{p}, \chi_{T}, s+\frac{1}{2}\right) I_{\Omega}(s) .
$$

$L\left(\pi_{p}, \chi_{T}, s+\frac{1}{2}\right)$ is the standard $L$-function of $\pi_{p}$ twisted by $\chi_{T}$, the quadratic character associated to $T$ (at the prime $p$ ). Thus

$$
I(s)=\lim _{\Omega} I_{\Omega}(s)=J(s) L_{S}\left(\pi, \chi_{T}, s+\frac{1}{2}\right)
$$

where

$$
L_{S}\left(\pi, \chi_{T}, s+\frac{1}{2}\right)=\prod_{p \notin S} L\left(\pi_{p}, \chi_{T}, s+\frac{1}{2}\right) .
$$

Next, it is possible to show that at the finite primes of $S$ one can choose $\phi$ and $f$ such that

$$
J(s)=\int_{N(\mathbf{R}) \backslash G(\mathbf{R})} \varphi_{T}(g) \omega(g) \phi_{\infty}\left(I_{n}\right) f_{\infty}(g, s) d g .
$$

By the Iwasawa decomposition $J(s)$ has the form

$$
\int_{K \infty} H(k, s) f_{\infty}(k, s) d k
$$


where

$$
H(k, s)=\int_{G L(n, \mathbf{R})} \varphi_{T}(g k) \omega(k) \phi_{\infty}(g)|\operatorname{det} g|^{s-1 / 2} \chi_{T}(\operatorname{det} g) d g
$$

( $K$ is the maximal compact subgroup of $G(\mathbf{A})$ ). Assuming that $\phi$ has the form $P(x) e^{-\pi \operatorname{tr}\left({ }^{\prime} x x\right)}$, where $P(x)$ is a polynomial in $x$ (this is a stable subspace of $\left(S(M(n, \mathbf{R}))\right.$, under $\left.\omega\left(K_{\infty}\right)\right)$, it is easy to see that the meromorphic continuation of $J(s)$ is determined by that of

$$
\int_{\mathrm{GL}(n, \mathbf{R})} \varphi_{T}(g) \phi_{\infty}(g)|\operatorname{det} g|^{s-1 / 2} \chi_{T}(\operatorname{det} g) d g
$$

and even simpler, using the Cartan decomposition for $\operatorname{GL}(n, \mathbf{R})$, it is enough to consider integrals of the form

$$
\int_{a_{2}, \ldots, a_{n} \geq 1, a_{1}>0} \lambda\left(\pi_{\infty}(a) v\right) \phi(a) a_{1}^{s_{1}} a_{2}^{s_{2}} \cdots a_{n}^{s_{n}} d^{*}\left(a_{1}, \ldots, a_{n}\right) .
$$

Here $a=\operatorname{diag}\left(a_{1} a_{2} \ldots a_{n}, a_{1} a_{2} \ldots a_{n-1}, \ldots, a_{1} a_{2}, a_{1}\right), v$ is a $K_{\infty}$-finite vector in the space of $\pi_{\infty}$. $\lambda$ is a linear functional obtained as follows. Fix a vector $v_{p}$ in the space of $\pi_{p}$ for all (finite) primes, so that $v_{p}$ is unramified for almost all $p$. Let $\varphi_{v}$ be the cusp form corresponding to $v \otimes\left(\otimes_{p} v_{p}\right)$. Let $\xi$ be a matrix coefficient on $K^{\prime}=K_{\infty} \cap \mathrm{GL}(n, \mathbf{R})$, then

$$
\lambda(v)=\int_{K^{\prime}}\left(\varphi_{\pi_{\infty}(u) v}\right)_{T}(I) \xi(u) d u,
$$

$\phi$ is in $S\left(\mathbf{R}^{n}\right)$.

The integral (A) converges absolutely for $\operatorname{Re}\left(s_{1}\right) \gg 0$ and all $s_{2}, \ldots, s_{n}$. To obtain meromorphic continuation in $s_{1}$, we use a variation on Wallach's method [W, Theorems 5.8 and 7.2]. For that we have to obtain an asymptotic expansion of $\lambda\left(\pi_{\infty}(a) v\right)$ in $a_{1}$, determining an explicit dependence of the expansion on $a_{2}, \ldots, a_{n}$. This is the main theorem of our paper. The basic property of $\lambda$ that we need is that of certain moderate growth which is analogous to (7.1) in [W]. The fact that the functional $\lambda$ in $(B)$ is defined through the Fourier coefficient $\varphi_{T}$ leads us to call the integrals in question "Whittaker integrals".

\section{Notation}

(a) $G=\underline{G}_{\mathbf{R}}$, the real points of a reductive group $\underline{G}$, defined over $\mathbf{R}$. We assume that $G={ }^{0} G=\bigcap \operatorname{Ker} \chi, \chi$ running over the continuous homomorphisms of $G$ into $\mathbf{R}^{*}$. Let $\mathscr{G}$ be the Lie algebra of $G$.

(b) $K$ is the maximal compact subgroup of $G$, corresponding to $k \subset \mathscr{G}$, the fixed point of $\theta$, a Cartan involution of $\mathscr{G}$. Consider the $(-1)$ eigenspace of $\theta$, and in it let $\mathscr{A}_{0}$ be the maximal subspace such that $\left[\mathscr{A}_{0}, \mathscr{A}_{0}\right]=0$.

(c) Let $\phi\left(\mathscr{G}, \mathscr{A}_{0}\right)$ be the set of roots of $\mathscr{G}$ relative to $\mathscr{A}_{0}, \phi^{+}\left(\mathscr{G}, \mathscr{A}_{0}\right)$-the positive roots (with respect to some fixed order). Let $\mathscr{N}_{0}$ be the subalgebra of $\mathscr{G}$, spanned by the positive roots spaces. Denote by $P_{0}$ the minimal parabolic subgroup of $G$ corresponding to $\mathscr{N}_{0} ; P_{0}=\left\{g \in G \mid \operatorname{Ad}(g) \mathscr{N}_{0} \subset \mathscr{N}_{0}\right\}$. The 
unipotent radical of $P_{0}$ is $N_{0}=\exp \mathscr{N}_{0} . A_{0}=\exp \mathscr{A}_{0}$ is the connected component of the center of $M_{0}$, the Levi part of $P_{0}$.

(d) $(P, A)$ denotes a standard parabolic pair. Thus $P$ is a subgroup of $G$, containing $P_{0}$. Let $P=M N$ be the Levi decomposition of $P$; then $A$ is the connected component of the center of the Levi part $M$. Let $\mathscr{A}, \mathscr{N} \subset \mathscr{G}$ be the Lie algebras of $A$ and $N$; then $\mathscr{N}=\bigoplus_{\gamma \in \mathscr{A}^{*}} \mathscr{N}_{\gamma}$, where $\mathscr{N}_{\gamma}=\{X \in$ $\mathscr{N} \mid[H, X]=\gamma(H) X, H \in \mathscr{A}\}$. Put $\phi(P, A)=\left\{\gamma \in \mathscr{A}^{*} \mid \mathscr{N}_{\gamma} \neq 0\right\}$. We have $\phi^{+}\left(\mathscr{G}, \mathscr{A}_{0}\right)=\phi\left(P_{0}, A_{0}\right)$ and $\left.\phi(P, A) \subset \phi\left(P_{0}, A_{0}\right)\right|_{\mathscr{A}}$.

\section{THE THEOREM}

2.1. Let $(P, A)$ be a standard parabolic pair, such that $\operatorname{dim} A=1$. Let $\left\{\alpha_{1}, \ldots\right.$, $\left.\alpha_{l}\right\}$ be the simple roots of $\phi\left(P_{0}, A_{0}\right)$, and let $H_{1}, \ldots, H_{l}$ in $\mathscr{A}_{0}$ satisfy $\alpha_{i}\left(H_{j}\right)=$ $\delta_{i j} ; i, j=1, \ldots, l$. We may assume that $\mathscr{A}=\mathbf{R} H_{1}$. Let $a_{t}=\exp \left(-t H_{1}\right)$ and $a^{\prime} \quad=$ $\exp \left(\sum_{i=2}^{i=l} t_{i} H_{i}\right)$ for $t, t_{i} \succeq 0$. Clearly, $a^{\prime}$ lies in the closure of $A_{0}^{+}$-the positive Weyl chamber, and $a^{\prime} \in \operatorname{Ker} \alpha_{1}$.

Let $V$ be a finitely generated admissible $(\mathscr{G}, K)$ module, and $(\pi, \mathscr{H})$ an admissible representation on a Hilbert space $\mathscr{H}$ such that $V$ is isomorphic to $\mathscr{H}_{K}$, the subspace of $K$-finite vectors of $\mathscr{H}$. We identify $V=\mathscr{H}_{K}$. Let $V_{1}$ be the $G$-module generated by $V$. Let $\lambda \in V_{1}^{*}$, the algebraic dual of $V_{1}$, satisfy the following conditions:

(1) For $v \in V$, the function $g \mapsto \lambda(\pi(g) v)$ is in $C^{\infty}(G)$, and for $g \in G$, $X \in \mathscr{G}$,

$$
\left.\frac{d}{d t}(\lambda(\pi(g \exp t X) v))\right|_{t=0}=\lambda(\pi(g)(X \cdot v)) .
$$

(2) There is a $\mu \in \mathbf{R}$, such that for $X \in \mathscr{U}(\mathcal{N})$ and $v \in V$, there is a polynomial $P_{X, n}$ in $l-1$ variables with positive coefficients satisfying

$$
\left|X \cdot \lambda\left(\pi\left(a_{t} a^{\prime}\right) v\right)\right| \leq P_{X, v}\left(e^{t_{2}}, \ldots, e^{t_{t}}\right) e^{\mu t} .
$$

We put $P_{X, v}\left(a^{\prime}\right)=P_{X, v}\left(e^{t_{2}}, \ldots, e^{t_{l}}\right)$.

Let $\phi_{P}^{\prime}$ be the subset of roots $\gamma$ in $\phi\left(P_{0}, A_{0}\right)$, such that the unipotent subgroup corresponding to $\gamma$ lies in $N$. Let $b$ be the maximal coefficient in the expression $\gamma=\sum_{i=2}^{l} n_{i} \alpha_{i}$, when $\gamma$ runs over $\phi_{p}^{\prime}$. Define

$$
L\left(a^{\prime}\right)=\left(\prod_{i=2}^{l} a^{\prime \alpha_{i}}\right)^{b}
$$

( $a^{\prime}$ is always in $\operatorname{Ker} \alpha_{1} \cap \overline{A_{0}^{+}}$). 
2.2 Example. Let $\underline{G}=\mathrm{Sp}(n)$, the symplectic group of rank $n$. Choose $P_{0}=B$, the standard Borel subgroup

$$
\left(\begin{array}{llllll}
* & & * & & & \\
& \ddots & & & * & \\
0 & & * & & & \\
& & & * & & 0 \\
& 0 & & & \ddots & \\
& & & * & & *
\end{array}\right)
$$

then

$$
A_{0}=\left\{a=\left(\begin{array}{cccccc}
x_{1} & & & & & \\
& \ddots & & & & \\
& & x_{n} & & & \\
& & & x_{1}^{-1} & & \\
& & & & \ddots & \\
& & & & & x_{n}^{-1}
\end{array}\right) \mid x_{1}, \ldots, x_{n}>0\right\} .
$$

The simple roots are $\alpha_{1}, \ldots, \alpha_{n}$ where $a^{\alpha_{i}}=x_{i-1} x_{i}^{-1}$ for $i=2, \ldots, n$ and $a^{\alpha_{1}}=x_{n}^{2}, a \in A_{0}$. Let $P=\left(\begin{array}{c}* * \\ 0 *\end{array}\right)$. Then

$$
\begin{gathered}
A=\left\{\left(\begin{array}{cc}
x I_{n} & \\
& x^{-1} I_{n}
\end{array}\right) \mid x>0\right\}, \quad \mathscr{A}=\left\{\left(\begin{array}{ll}
z I_{n} & \\
& -z I_{n}
\end{array}\right) \mid z \in \mathbf{R}\right\}, \\
\mathscr{N}=\left\{\left.\left(\begin{array}{ll}
0 & x \\
0 & 0
\end{array}\right)\right|^{t_{x}}=x \in M(n, \mathbf{R})\right\} .
\end{gathered}
$$

Take

$$
H_{1}=\frac{1}{2}\left(\begin{array}{ll}
I_{n} & \\
& -I_{n}
\end{array}\right), \quad H_{j}=\left(\begin{array}{ll}
E_{j} & \\
& -E_{j}
\end{array}\right), \quad j=2, \ldots, n,
$$

where

$$
E_{j}=\left(\begin{array}{cccc}
I_{j-1} & & & \\
& 0 & & \\
& & \ddots & \\
& & & 0
\end{array}\right) .
$$

Clearly $\alpha_{i}\left(H_{j}\right)=\delta_{i j}$. We have

$$
a_{t}=\left(\begin{array}{cc}
e^{-t / 2} I_{n} & \\
& e^{-t-2} I_{n}
\end{array}\right), \quad t \geq 0
$$


and

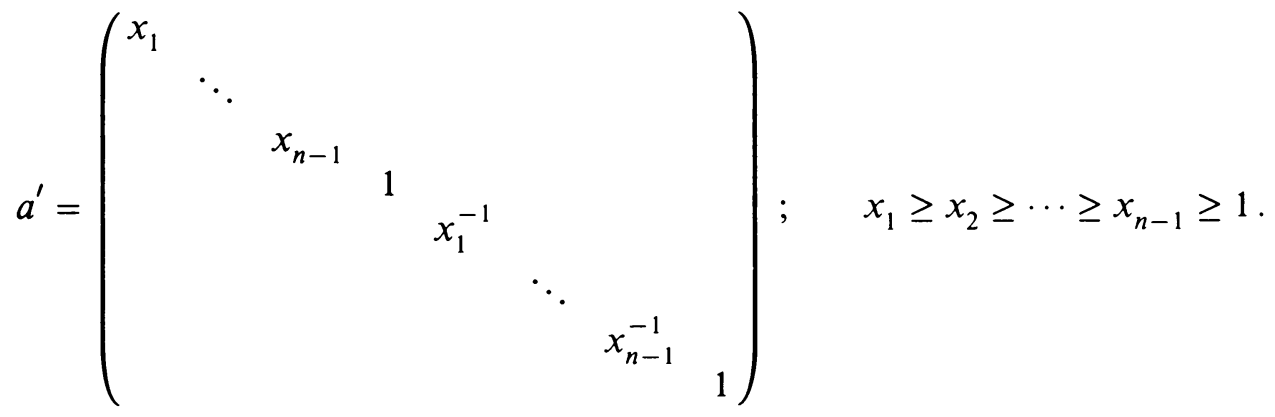

Let $V$ be the space of $K$-finite vectors for a representation $\pi$, which is a local component of an irreducible, automorphic cuspidal representation $\tau$ of $\operatorname{Sp}(n, \mathbf{A})$, A being the ring of adeles of $\mathbf{Q}$. Let $\psi$ be a nontrivial character of $\mathbf{Q} \backslash \mathbf{A}$ and $T$ a symmetric matrix in $M(n, \mathbf{Q})$. For a cusp form $\varphi$ in $T$, consider the Fourier coefficient

$$
\varphi_{T}(g)=\int_{N_{\mathbf{Q} \backslash N_{\mathrm{A}}}} \psi^{-1}(\operatorname{tr} T X) \varphi\left(\left(\begin{array}{cc}
I_{n} & X \\
0 & I_{n}
\end{array}\right) g\right) d x
$$

Fix a vector $v_{p}$ in the space of $\tau_{p}$ for $p$-prime, so that $v_{p}$ is unramified for almost all $p$. Let $\varphi_{v}$ be the cusp form corresponding to $v \oplus\left(\bigoplus_{p} v_{p}\right)$, where $v \in V$. Define $\bigwedge(v)=\left(\varphi_{v}\right)_{T}(I)$, for $v \in V$. $\bigwedge$ certainly satisfies the conditions (1), (2) in 2.1. Denote $K^{\prime}=K \cap M,(P=M N)$, and let $w(u)$ be a matrix coefficient on $K^{\prime}$. Put,

$$
\lambda(v)=\int_{K^{\prime}} \bigwedge(\pi(u) v) w(u) d u .
$$

It is easy to see that $\lambda$ satisfies the conditions (1), (2) as well. (Actually $\bigwedge(\pi(g) v)$ and $\lambda(\pi(g) v)$ are bounded functions of $g$ in $G)$.

2.3. Put $H=-H_{1}$. We are at the situation of $\S 7.2$ of [W], where an asymptotic expansion is derived for $\lambda\left(\pi\left(a_{t}\right) v\right)$ as $t$ tends to infinity. Our task is to estimate the polynomials $p_{i, n}(t, v)$ of the expansion when $v$ is replaced by $\pi\left(a^{\prime}\right) v$. So, we also consider the finite set $E_{k}(P, V)$ of the eigenvalues of $H$ acting on the finitely generated admissible space $V / \mathscr{N}^{k} V$. For $\xi \in$ $E_{k}(P, V), H-\xi$ has a bounded degree $d$ of nilpotence on $V / \mathscr{N}^{k} V$. We have

$$
E_{k+1}(P, V)-E_{k}(P, V) \subset\left\{\xi-\alpha\left(H_{1}\right) \mid \xi \in E_{k}(P, V), \alpha \in \phi(P, A)\right\}
$$

and so $\bigcup_{k=1}^{\infty} E_{k}(P, V) \subset\left\{\xi-n \mid \xi \in E_{1}(P, V), n=0,1,2, \ldots\right\}$. Let $z_{1}, \ldots$, $z_{e} \in E_{1}(P, V)$ be such that if $z_{i}-z_{j}$ is a nonzero integer, then $i=j$, and such that every $\xi$ in $E_{1}(P, V)$ has the form $z_{i}-n, n=0,1, \ldots$. Arrange $\left\{z_{i}-n \mid 1 \leq i \leq e, \quad 0 \leq n \in \mathbf{Z}\right\}=\left\{\xi_{1}, \xi_{2}, \ldots\right\}$ so that $\operatorname{Re} \xi_{1} \geq \operatorname{Re} \xi_{2} \geq \cdots$. Choose the indices $1 \leq N_{1}<N_{2}<\cdots$ as follows: $N_{1}+1$ is the first such that $\operatorname{Re} \xi_{N_{1}+1}<\operatorname{Re} \xi_{1}, N_{2}+1$ is the first such that $\operatorname{Re} \xi_{N_{2}+1}<\operatorname{Re} \xi_{N_{1}+1}$ etc. 
2.4 Theorem. Let $\xi=\xi_{N_{i}}, i \geq 1$. Let $k=k_{N_{i}} \geq 1$ be an integer such that $-k+\mu<\operatorname{Re} \xi-1$. Then there exist $\xi_{r_{1}}, \ldots, \xi_{r_{2}}$ depending on $v$ and $k$, and there exist polynomials in $t, P_{\xi_{j}, k}\left(t, a^{\prime}, v\right)$ with coefficients bounded by $P_{k, v}^{*}\left(a^{\prime}\right) L^{k}\left(a^{\prime}\right)$, where $P_{k, v}^{*}\left(a^{\prime}\right)$ is a polynomial function with positive coefficients depending on $k, v$, and such that for $t \geq 0$ and $a^{\prime} \in \operatorname{Ker} \alpha_{1} \cap \overline{A_{0}^{+}}$,

$$
\left|\lambda\left(\pi\left(a_{t} a^{\prime}\right) v\right)-\sum_{j=1}^{s} e^{t \xi_{r_{j}}} P_{\xi_{j, k}}\left(t, a^{\prime}, v\right)\right| \leq e^{t \operatorname{Re} \xi} P_{k, v}\left(a^{\prime}\right) L^{k}\left(a^{\prime}\right)
$$

where $P_{k, v}\left(a^{\prime}\right)$ is a polynomial function with positive coefficients depending on $k, v$.

Proof. Assume first that $v \in \mathscr{N}^{k} V$, there is a polynomial $P_{k, v}\left(a^{\prime}\right)$ with positive coefficients such that

$$
\left|\lambda\left(\pi\left(a_{t} a^{\prime}\right) v\right)\right|<e^{(\operatorname{Re} \xi-1) t} L^{k}\left(a^{\prime}\right) P_{k, v}\left(a^{\prime}\right) .
$$

Let $X_{1}, \ldots, X_{m}$ be a basis of $\mathscr{N}$ such that $\left[\tilde{H}, X_{1}\right]=\gamma_{i}(\tilde{H}) X_{i}$ for $\tilde{H}$ in $\mathscr{A}_{0}$. Note that for $\tilde{H}=H, \gamma_{i}(\tilde{H}) \leq-1$. Write $v=\sum X_{i_{1}} \cdots X_{i_{k}} v_{i_{1}} \cdots_{i_{k}}$, then

$$
\begin{aligned}
\left|\lambda\left(\pi\left(a_{t} a^{\prime}\right) v\right)\right| & \leq \sum\left|\lambda\left(\operatorname{Ad}\left(a_{t} a^{\prime}\right) X_{i_{1}} \cdots \operatorname{Ad}\left(a_{t} a^{\prime}\right) X_{i_{k}} \cdot \pi\left(a_{t} a^{\prime}\right) v_{i_{1}} \cdots i_{k}\right)\right| \\
& \leq e^{-k t} L^{k}\left(a^{\prime}\right) \sum P_{X_{i_{1}} \cdots X_{i_{k}}, v_{i_{1}} \cdots i_{k}}\left(a^{\prime}\right) e^{\mu t}=e^{(\mu-k) t} L^{k}\left(a^{\prime}\right) P_{k, v}\left(a^{\prime}\right) \\
& <e^{(\operatorname{Re} \xi-1) t} L^{k}\left(a^{\prime}\right) P_{k, v}\left(a^{\prime}\right) .
\end{aligned}
$$

From now on $P_{k, v}\left(a^{\prime}\right), P_{k, v}^{(1)}\left(a^{\prime}\right), \tilde{P}_{k, v}\left(a^{\prime}\right)$ etc. will always denote a polynomial function with positive coefficients. Now assume that $v \notin \mathscr{N}^{k} V$. Let $q_{k}: V \rightarrow$ $V / \mathscr{N}^{k} V$ be the natural projection. Let $v_{1}=v$ and $\bar{v}_{1}=q_{k}\left(v_{1}\right), \ldots, \bar{v}_{n}=$ $q_{k}\left(v_{n}\right)$ be a basis for $\mathscr{U}(\mathscr{A}) v_{1}$, and let $B=\left(b_{i j}\right)$ be the matrix of $H$ such that $H \cdot v_{i}=\sum_{j=1}^{n} b_{i j} v_{j}$. The eigenvalues of $B$ are contained in $E_{k}(P, V)$. We have

$$
H \cdot v_{i}=\sum_{j=1}^{n} b_{i j} v_{j}+w_{i}, \quad w_{i} \in \mathscr{N}^{k} V
$$

So, putting

$$
F\left(t, a^{\prime}, v\right)=\left(\begin{array}{c}
\lambda\left(\pi\left(a_{t} a^{\prime}\right) v_{1}\right) \\
\vdots \\
\lambda\left(\pi\left(a_{t} a^{\prime}\right) v_{n}\right)
\end{array}\right), \quad G\left(t, a^{\prime}, v\right)=\left(\begin{array}{c}
\lambda\left(\pi\left(a_{t} a^{\prime}\right) w_{1}\right) \\
\vdots \\
\lambda\left(\pi\left(a_{t} a^{\prime}\right) w_{n}\right)
\end{array}\right)
$$

we have

Solving, we get

$$
\frac{d}{d t} F\left(t, a^{\prime}, v\right)=B F\left(t, a^{\prime}, v\right)+G\left(t, a^{\prime}, v\right) .
$$

$$
F\left(t, a^{\prime}, v\right)=e^{t B} F\left(0, a^{\prime}, v\right)+e^{t B} \int_{0}^{t} e^{-\tau B} G\left(\tau, a^{\prime}, v\right) d \tau
$$


Let $\xi_{r_{1}}, \xi_{r_{2}}, \ldots, \xi_{r_{s}}$ be the eigenvalues of $B$ on $\mathbf{C}^{n}$ arranged such that $\operatorname{Re} \xi_{r_{1}} \geq \operatorname{Re} \xi_{r_{2}} \geq \cdots \geq \operatorname{Re} \xi_{r_{s}}$. Let $P_{j}$ be the projection of $\mathbf{C}^{n}$ on the $\xi_{r_{j}}$ generalized eigenspace. Put $Q=\sum_{r_{j}>N_{i}} P_{j}$ and $R=\sum_{r_{j} \leq N_{i}} P_{j}, Q+R=I_{n}$. We first estimate that there is $\tilde{P}_{k, v}\left(a^{\prime}\right)$ such that

$$
\left\|Q\left(F\left(t, a^{\prime}, v\right)\right)\right\| \leq e^{t \operatorname{Re} \xi} \tilde{P}_{k, v}\left(a^{\prime}\right) L^{k}\left(a^{\prime}\right) .
$$

Let $u \in \mathbf{C}^{n}$. Since $P_{j}$ commutes with $B$, we have

$$
\left\|P_{j}\left(e^{t B}(u)\right)\right\|=\left\|e^{t B}\left(P_{j}(u)\right)\right\| \leq P_{B}(t) e^{t \operatorname{Re} \xi_{r_{j}}}\|u\|,
$$

$P_{B}(t)$ is a fixed polynomial depending on $B$ (and hence on $v$ ). Since, for $r_{j}>N_{i}$, we have $\operatorname{Re} \xi_{r_{j}}<\operatorname{Re} \xi,\left(\xi=\xi_{N_{i}}\right)$, then there is a constant $C_{k, v}$ such that $\sum_{r_{j}>N_{i}} P_{B}(t) e^{t \operatorname{Re} \xi_{r_{j}}} \leq C_{k, v} e^{t \operatorname{Re} \xi}$. Thus, letting $\left\{e_{1}, \ldots, e_{n}\right\}$ be the standard basis of $\mathbf{C}^{n}$, we get

$$
\begin{aligned}
\left\|Q\left(e^{t B} F\left(0, a^{\prime}, v\right)\right)\right\| & \leq \sum_{\nu=1}^{n}\left|\lambda,\left(\pi\left(a^{\prime}\right) v_{\nu}\right)\right|\left\|Q\left(e^{t B}\left(e_{\nu}\right)\right)\right\| \\
& \leq C_{k, v} \sum_{\nu=1}^{n} P_{k, v_{\nu}}\left(a^{\prime}\right) e^{t \operatorname{Re} \xi} \leq e^{t \operatorname{Re} \xi} P_{k, v}^{(1)}\left(a^{\prime}\right) \\
& \leq e^{t \operatorname{Re} \xi} P_{k, v}^{(1)}\left(a^{\prime}\right) L^{k}\left(a^{\prime}\right)
\end{aligned}
$$

$\left(P_{k, v_{\nu}}\left(a^{\prime}\right)\right.$ satisfies $\left.\left|\lambda\left(\pi\left(a^{\prime}\right) v_{\nu}\right)\right| \leq P_{k, v_{\nu}}\left(a^{\prime}\right)\right)$. Similarly, using (1) and the fact that $w_{\nu} \in \mathcal{N}^{k} V$, we find that there is $P_{k, v}^{(2)}\left(a^{\prime}\right)$ such that

$$
\left\|Q\left(e^{t B} \int_{0}^{t} e^{-\tau B} G\left(\tau, a^{\prime}, v\right) d \tau\right)\right\| \leq e^{t \operatorname{Re} \xi} P_{k, v}^{(2)}\left(a^{\prime}\right) L^{k}\left(a^{\prime}\right) .
$$

This proves (3). Now let $r_{j} \leq N_{i}$. Note that $\operatorname{Re} \xi_{r_{j}} \geq \operatorname{Re} \xi$. As before, there is a polynomial $P_{k, v}^{(3)}\left(a^{\prime}\right)$ such that

$$
\begin{aligned}
\left\|P_{j}\left(e^{-\tau B} G\left(\tau, a^{\prime}, v\right)\right)\right\| & \leq e^{(\mu-k-\operatorname{Re} \xi) \tau} P_{k, v}^{(3)}\left(a^{\prime}\right) L^{k}\left(a^{\prime}\right) \\
& <e^{-\tau} P_{k, v}^{(3)}\left(a^{\prime}\right) L^{k}\left(a^{\prime}\right) .
\end{aligned}
$$

This shows that $I\left(a^{\prime}, v\right)=\int_{0}^{\infty} R\left(e^{-\tau B} G\left(\tau, a^{\prime}, v\right)\right) d \tau$ converges absolutely. Put $F^{\circ}\left(t, a^{\prime}, v\right)=R\left(e^{t B}\left(F\left(0, a^{\prime}, v\right)+I\left(a^{\prime}, v\right)\right)\right)$, then as before,

$$
\begin{aligned}
& \left\|R\left(F\left(t, a^{\prime}, v\right)\right)-F^{\circ}\left(t, a^{\prime}, v\right)\right\| \leq L^{k}\left(a^{\prime}\right) P_{k, v}^{(4)}\left(a^{\prime}\right) P_{B}(t) \\
& \quad \times \sum_{r_{j} \leq N_{i}} e^{t \operatorname{Re} \xi_{r_{j}}} \int_{t}^{\infty} P_{B}(\tau) e^{\left(\mu-k-\operatorname{Re} \xi_{r_{j}}\right) \tau} d \tau \\
& \leq c \cdot L^{k}\left(a^{\prime}\right) P_{k, v}^{(4)}\left(a^{\prime}\right) P_{B}(t) \sum_{r_{j} \leq N_{i}} e^{t \operatorname{Re} \xi_{r_{j}}} \int_{t}^{\infty} e^{\left(u-k-\operatorname{Re} \xi_{r_{j}}+1\right) \tau} d \tau \\
& =L^{k}\left(a^{\prime}\right) P_{k, v}^{(5)}\left(a^{\prime}\right) e^{(\mu-k+1) t}<L^{k}\left(a^{\prime}\right) P_{k, v}^{(5)}\left(a^{\prime}\right) e^{t \operatorname{Re} \xi} .
\end{aligned}
$$


This last estimate and (3) show that there is a polynomial $P_{k, v}\left(a^{\prime}\right)$ such that

$$
\left\|F\left(t, a^{\prime}, v\right)-F^{\circ}\left(t, a^{\prime}, v\right)\right\| \leq e^{t \operatorname{Re} \xi} P_{k, v}\left(a^{\prime}\right) L^{k}\left(a^{\prime}\right)
$$

$(\operatorname{Re} \xi$ could be replaced by $\operatorname{Re} \xi-\varepsilon$ for some $\varepsilon>0)$.

Let $\psi\left(t, a^{\prime}, v\right)$ be the first coordinate of $F^{\circ}\left(t, a^{\prime}, v\right)$; then (4) implies that for $v \notin \mathscr{N}^{k} V$

$$
\left|\lambda\left(\pi\left(a_{t} a^{\prime}\right) v\right)-\psi\left(t, a^{\prime}, v\right)\right| \leq e^{t \operatorname{Re} \xi} P_{k, v}\left(a^{\prime}\right) L^{k}\left(a^{\prime}\right) .
$$

Extend $\psi\left(t, a^{\prime}, v\right)$ to be zero on $\mathcal{N}^{k} V$, and (5) is still true because of (1). It remains to note that by definition

$$
\psi\left(t, a^{\prime}, v\right)=\sum_{j=1}^{s} e^{t \xi_{r_{j}}} P_{\xi_{r_{j}}}, k\left(t, a^{\prime}, v\right)
$$

where $P_{\xi_{r_{j}, i}}\left(t, a^{\prime}, v\right)$ are polynomials in $t$ (zero polynomials for $v \in \mathscr{N}^{k} V$ ), with coefficients $\beta\left(a^{\prime}, v\right)$ satisfying $\left|\beta\left(a^{\prime}, v\right)\right| \leq P_{k, v}^{*}\left(a^{\prime}\right) L^{k}\left(a^{\prime}\right)$ for some polynomial function $P_{k, v}^{*}$ on $\operatorname{Ker} \alpha_{1} \cap \overline{A_{0}^{+}}$.

2.5 Corollary. Assume that $\lambda$ satisfies a similar condition to (2) of 2.1 also for $t<0$. Let $\phi \in S\left(\mathbf{R}^{l}\right)$, the Schwartz-Bruhat functions on $\mathbb{R}^{i}$, then

$$
\int_{a^{\prime} \in \overline{A_{0}^{+}}} \int_{-\infty}^{\infty} \lambda\left(\pi\left(a_{t} a^{\prime}\right) v\right) \phi\left(e^{-t}, a^{\prime \alpha_{2}}, \ldots, a^{\prime \alpha_{l}}\right) e^{-s_{1} t} \cdots\left(a^{\prime \alpha_{l}}\right)^{s_{l}} d t d a^{\prime}
$$

converges for $\operatorname{Re}\left(s_{1}\right) \gg 0$ and all $s_{2}, \ldots, s_{l}$ and it has a meromorphic continuation in $s_{1}$.

Proof. For the integration over $0<t$ apply (5) and (6) of the proof in (2.4). For the integration over $t<0$, there is no problem of convergence by our assumption on $\lambda$ and the presence of $\phi$ in the integral.

2.6. Going to the example explained in the Introduction, we obtain (using the notation there)

Corollary. The integral

$$
\int_{\mathrm{GL}(n, \mathbf{R})} \varphi_{T}(g) \phi_{\infty}(g)|\operatorname{det} g|^{s-1 / 2} \chi_{T}(\operatorname{det} g) d g
$$

(which converges absolutely for $\operatorname{Re}(s) \gg 0$ ) admits a meromorphic continuation to the whole plane.

\section{REFERENCES}

[A] A. N. Andrianov, On Zeta functions of Rankin type associated with Siegel modular forms. Lecture Notes in Math., vol. 627, Springer-Verlag, Berlin, Heidelberg and New York, 1977.

[PS-R] I. Piatetski-Shapiro and S. Rallis, A new way to get Euler $F$ oducts, Crelle Journal (to appear). 
[W] N. R. Wallach, Asymptotic expansions of generalized matrix entries of representations of real reductive groups, Lecture Notes in Math., vol. 1024, Springer-Verlag, Berlin, Heidelberg and New York, 1983.

Department of Mathematics, Ohio State University, Columbus, Ohio 43210-1174

Department of Mathematics, Tel Aviv University, IsReal 